The following paper posted here is not the official IEEE published version. The final published version of this paper can be found in the Proceedings of the Conference on Optical Fiber Communication (2006 : Anaheim, Calif.):3pp.

Copyright @ 2006 IEEE.

Personal use of this material is permitted. However, permission to reprint/republish this material for advertising or promotional purposes or for creating new collective works for resale or redistribution to servers or lists, or to reuse any copyrighted component of this work in other works must be obtained from the IEEE. 


\title{
Brillouin suppression through longitudinal structural variation in high nonlinearity silica holey fibers
}

\author{
F. Poletti, K. Furusawa, Z. Yussoff, P. Petropoulos, N. G. R. Broderick , T. M. Monro and D. J. Richardson \\ Optoelectronics Research Centre, University of Southampton, Southampton, SO17 1BJ, United Kingdom, \\ Email:frap@orc.soton.ac.uk
}

\begin{abstract}
We consider longitudinal variation in the fiber structure as a method of increasing the Brillouin linewidth and threshold within high nonlinearity silica holey fibers. Strategies to control the associated variation in nonlinearity and dispersion along the fiber length are described.

(C)2006 Optical Society of America

OCIS codes: (060.4370) Nonlinear Optics, fibers; (290.5900) Scattering, stimulated Brillouin;
\end{abstract}

\section{Introduction}

Holey optical fibers (HFs) can exhibit a variety of unusual optical properties that cannot be accessed using conventional optical fiber technology. Arguably, one of the most exciting possibilities is the potential for fibers with large values of Kerr nonlinearity per unit length with tailored dispersion characteristics, as required for applications such as optical switching, supercontinuum generation and wavelength conversion.

Whilst the Kerr nonlinearity and dispersion characteristics of a fiber are the primary issues for many optical signal processing applications, for others, particularly those employing narrow linewidth optical signals, the stimulated Brillouin scattering (SBS) properties of the fiber are also of great concern. Parasitic SBS can adversely affect the performance of Kerr based devices, limiting the maximum powers that can be used. The development of techniques that allow the relative strengths of these (often competitive) nonlinear processes within fiber to be controlled are thus extremely important. One possibility for controlling the effective strength of the Brillouin effect is to change the fiber structural parameters along its length in order to vary the local Brillouin linewidth down the fiber [1]. This has the effect of broadening the effective Brillouin linewidth of the fiber as a whole, thereby reducing the overall effective gain coefficient and increasing the SBS threshold. This has been shown to work well in conventional fibers where SBS suppression factors in excess of $7 \mathrm{~dB}$ have previously been reported [1]. Broadening of the Brillouin linewidth (by a factor of 7 or so) due to structural variation along a length of highly nonlinear silica HF has previously been reported [2]. However, in this previous work there was no attempt to correlate the observed line broadening with the structural variation along the fiber length, nor any attempt to establish how modification of the structure affected the other linear/nonlinear properties of the fiber. This is a key issue if such fibers are to be used and optimized for nonlinear device applications.

In this paper we present the results of systematic measurements of the Brillouin frequency shift (BFS) as a function of structure within a small core holey fiber which confirm that BFS of a HF scales linearly to first order with respect to the effective index, as would be expected from a simple consideration of the SBS process. We then present the results of a numerical study which shows how the corresponding linear and nonlinear waveguide properties change with structure for a hexagonally stacked silica HF, and identify several longitudinal fiber structural modulation strategies designed to control the dispersion variation in high nonlinearity HFs with high SBS threshold.

\section{Results and discussion}

We first produced a $40 \mathrm{~m}$ length of small-core, hexagonally-stacked, silica $\mathrm{HF}$ with deliberate longitudinal structural variation along its length (affected by making a step-change to the fiber draw speed during the drawing process). We then cut up this length of fiber into $2 \mathrm{~m}$ section and measured both the hole-to-hole spacing $(\Lambda)$ and the relative hole diameter $(\mathrm{d} / \Lambda)$ from SEM images of the samples, and then measured the BFS associated with each sample [3]. The variation in structural parameters along the fiber length and a typical SEM image are shown in Fig.1. In Fig.2 we plot the measured BFS and the modeled effective-index along the fiber length (calculated using a finite element technique from the structural parameters taken form the SEM profiles of each of the $2 \mathrm{~m}$ samples). A direct correlation is observed between the effective index and measured Brillouin shift in agreement with the simple scaling rule [4]:

$$
v_{B}(z)=\frac{2 n_{e f f}(z) \cdot v_{A}}{\lambda}
$$


where $v_{A}$ is the acoustic velocity within the fiber, $n_{\text {eff }}(z)$ the effective modal index, and $\lambda$ the vacuum wavelength of the pump lightwave. From our data $v_{A}=5500 \mathrm{~m} / \mathrm{s}$ in good agreement with previously reported values for germanium doped silica.

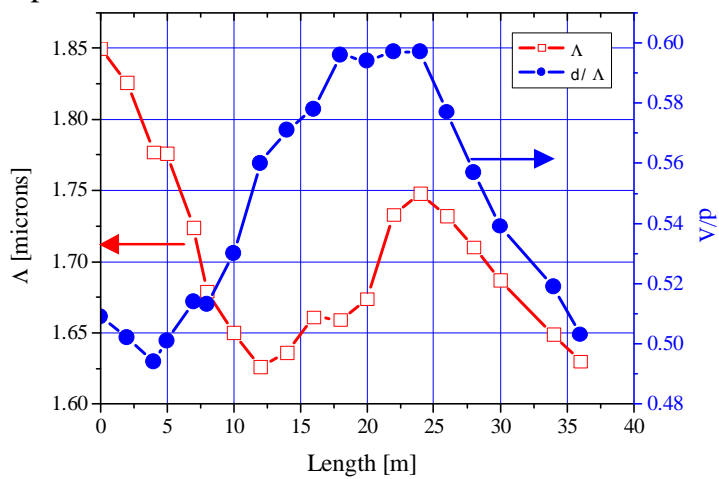

Fig. 1. Variation of the structural parameters $\Lambda$ and $\mathrm{d} / \Lambda$ along the length of the fabricated HF.

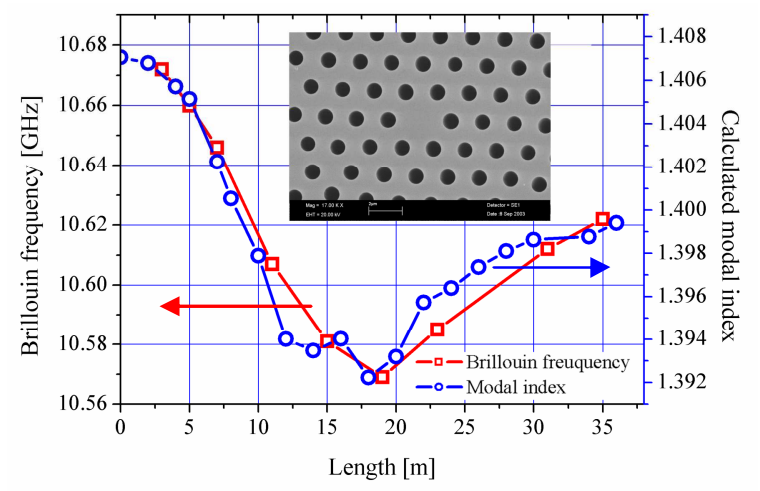

Fig.2. Comparison between the measured Brillouin shift and the simulated $\mathrm{n}_{\mathrm{eff}}$. Inset: a typical SEM image for this fiber.

It is to be appreciated that HF technology allows for a far greater range of $n_{\text {eff }}$ values than conventional doped fiber technology due to the large index contrast between glass and air, and thus offers great scope for significant Brillouin line broadening control. However, the other linear and nonlinear characteristics of HF are also highly sensitive to fiber structure and in order to understand the levels of sensitivity involved we undertook a detailed study

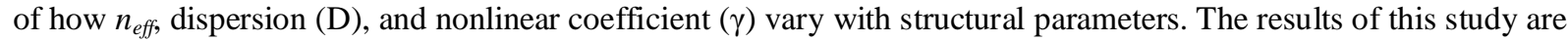
shown in Fig. 3 where we present multiple bi-dimensional contour plots showing the variation of these parameters over the range of $\Lambda$ and $\mathrm{d} / \Lambda$ defining small core, high nonlinearity HFs. As can be seen, $n_{\text {eff }}$ variation in the range 1.41 to 1.31 is obtainable - corresponding to a $7 \%$ variation in effective BFS across the plot. Note that the ratio of Brillouin width to BFS is typically $3.5 \times 10^{-3}$ for fused silica. Each $n_{\text {eff }}$ contour $(0.01)$ is thus seen to correspond to a shift of $\sim 2$ Brillouin linewidths. Superposed on the plot in Fig.3 and labeled as HF1 is the structural variation along the length of our fabricated fiber (every $2 \mathrm{~m}$ from the Beginning of Pull (BOP) to the End of Pull (EOP)). The effective index of the fiber is seen to vary between 1.407 to 1.392 over its length, resulting in an SBS shift variation of $\sim 3$ linewidths $(\sim 105 \mathrm{MHz})$, and an anticipated increase in SBS threshold by a factor of 2.2. From this plot it is seen that the fiber dispersion varies strongly along the length of the pull - ranging from $50 \mathrm{ps} / \mathrm{nm} / \mathrm{km}$ at the BOP, to $80 \mathrm{ps} / \mathrm{nm} / \mathrm{km}$ at the centre of the pull before dropping to $10 \mathrm{ps} / \mathrm{nm} / \mathrm{km}$ at the EOP. The dispersion slope varies between -0.1 and $-0.2 \mathrm{ps} / \mathrm{nm} / \mathrm{km}^{2}$ and the effective area by $\sim 30 \%$, from 5.9 to $4.2 \mu \mathrm{m}^{2}$. The increased Brillouin threshold is thus seen to be accompanied by very significant variations in key fiber properties for this particular structural modulation profile. Whilst these results highlight the sensitivities involved, this fiber was not designed a priori with SBS threshold reduction in mind, and the key issue is whether we can envisage modulation profiles for which substantial increases in SBS threshold can be achieved without associated large changes in the fiber dispersion properties in regimes of technological interest - e.g. for low values of dispersion. We propose two possible approaches which we also illustrate in Fig.3.

According to the first strategy we keep the dispersion constant by controllably modulating the fiber structure along the $\mathrm{D}=0$ contour (obviously this can be done for any other dispersion, or other fiber property contour, that one is interested in). The $n_{\text {eff }}$ contour density is highest in the small core, large air fill fraction region and thus substantial linewidth broadening and increased Brillouin thresholds should be achievable in this design space regime. For example, the fiber which we denoted $\mathrm{HF} 2$ has BOP $(\mathrm{d} / \Lambda, \Lambda)$ coordinates of $(0.48,1.62 \mu \mathrm{m})$ and EOP coordinates of $(0.68,1.35 \mu \mathrm{m})$, and closely follows the zero dispersion contour line. Assuming the fiber has a Brillouin gain spectrum with a Lorentzian profile $g_{b}(v, z)$ centered at $v_{b}(z)$ at each point $z$ along the length, we can calculate an effective gain coefficient as:

$$
G(v)=\int_{0}^{L} g_{b}(v, z) \cdot e^{-\alpha \cdot z} d z
$$

where $\alpha$ is the fiber loss and L its length [1]. The SBS threshold is ultimately inversely proportional to the maximum value of $\mathrm{G}(\mathrm{v})$, and therefore we can estimate the threshold increase resulting from structural non-uniformities as the ratio between the maximum gain coefficients of a uniform fiber and the fiber under analysis. We calculated that in the case of HF2 (assuming an intrinsic Brillouin gain bandwidth of $35 \mathrm{MHz}$, a typical attenuation of $0.1 \mathrm{~dB} / \mathrm{m}$ and a fiber length of $40 \mathrm{~m}$ ), the large difference in effective index, between 1.4 and 1.36 , would result in an overall 
linewidth of $260 \mathrm{MHz}(\sim 7.5$ times enhancement) and an increase in the SBS threshold of $\sim 4.7$ times $(6.7 \mathrm{~dB})$. HF2 would have an average nonlinear coefficient of $22 \mathrm{~W}^{-1} \mathrm{~km}^{-1}$ and zero dispersion at $1550 \mathrm{~nm}$ along its length.

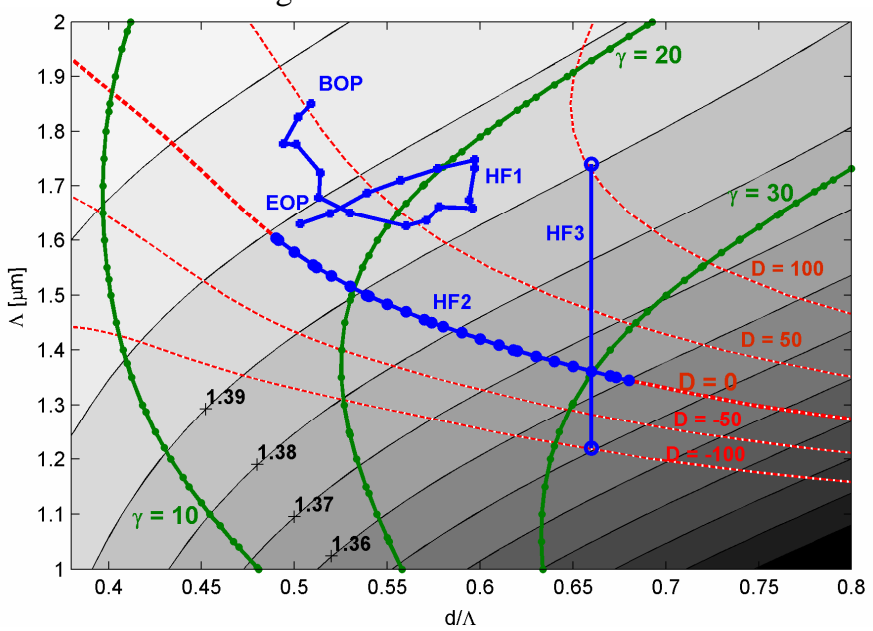

Fig.3. Contour plots showing the evolution of $\mathrm{n}_{\mathrm{eff}}$ (shaded in grayscale, every 0.01 ), D in $\mathrm{ps} / \mathrm{nm} / \mathrm{km}$ (dotted) and $\gamma$ in $\mathrm{W}^{-1} \mathrm{~km}^{-1}$ (small round markers) as a function of the structural parameters of a silica $\mathrm{HF}$ at $1550 \mathrm{~nm} . \mathrm{HF} 1$ is the trajectory of the fabricated HF, while HF2 and HF3 are two proposed examples.

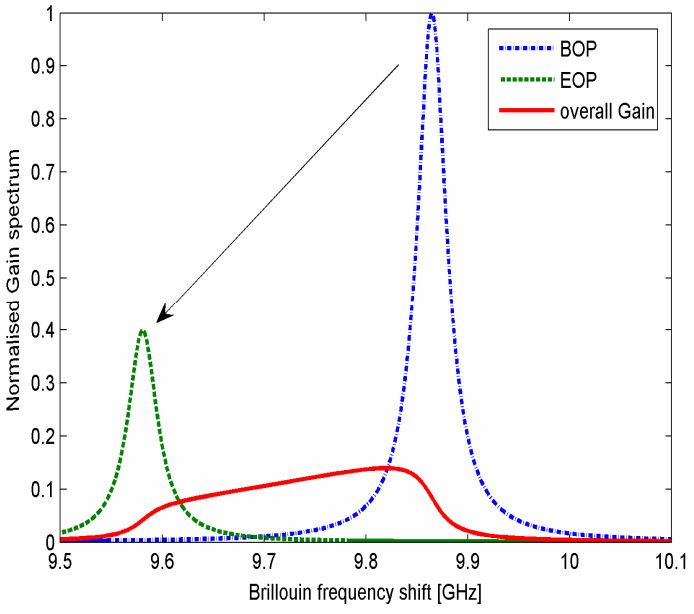

Fig.4. Simulated lineshape of the normalized Brillouin peak at BOP (dashed) and EOP peak (dotted) for the example in HF3. A fiber length of $40 \mathrm{~m}$ and a loss of 0.1 $\mathrm{dB} / \mathrm{m}$ are assumed. The continuous line represents the effective gain coefficient for the whole fiber.

The practical realization of such a fiber may however prove challenging since it requires accurate control and variation of two fiber parameters. A second strategy would be to target linear or cyclic trajectories within the structure design-space for which the ratio $\mathrm{d} / \Lambda$ is fixed and in which, by modifying only $\Lambda$, many effective index contours are crossed and at the same time a low path average dispersion is obtained. For example, the fiber following the trajectory labeled HF3, with a BOP of $(0.66,1.75 \mu \mathrm{m})$ and EOP of $(0.66,1.22 \mu \mathrm{m})$ would still have an $\mathrm{n}_{\mathrm{eff}}$ variation as large as 0.04 and provide a similar threshold increase as HF2 (Fig.4), yet with clear advantages in terms of ease of fabrication. This trajectory has been chosen in order to provide a low overall dispersion, averaged between the two extremes of $\pm 100 \mathrm{ps} / \mathrm{nm} / \mathrm{km}$, and at the same time a high average $\gamma$ of $\sim 28 \mathrm{~W}^{-1} \mathrm{~km}^{-1}$.

Both the proposed fibers HF2 and HF3 lie in a region of the structural design plane where the dispersion slope is between -0.1 and $-0.3 \mathrm{ps} / \mathrm{nm} / \mathrm{km}^{2}$. A similar design approach could be employed to target fibers with smaller dispersion slope, even if the nonlinearity has to be traded off. Alternatively, in order to minimize the extent of structural variation necessary, other trajectories which are at all points in a direction normal to the $n_{\text {eff }}$ contours can be envisaged.

\section{Conclusions}

In conclusion, we have demonstrated that HF technology provides great scope for managing the Brillouin lineshape (and effective Brillouin threshold) for high nonlinearity silica fibers due to the high index contrast between glass and air, allowing one to control the effective strength of Kerr and Brillouin nonlinearities through longitudinal manipulation of the structural parameters of the fiber. We have also shown that the dispersive and effective area properties of HF are also a very strong function of the fiber structure and that attention needs to be paid to how any structural modulation applied to increase the SBS threshold impacts the variation of these parameters. Finally, we have proposed modulation strategies to help manage the extent of dispersion variation within nonlinear fibers. It is to be appreciated that the techniques and strategies we have outlined here are applicable to a far broader range of fiber structures, operating wavelengths and indeed glass materials than we have presented. Note in particular that the relative size of the nonlinear Kerr and Brillouin coefficients themselves vary between glass types [5], which also offers additional advantage for Kerr based device applications involving narrow bandwidth signals.

\section{References}

[1] K. Shiraki et al., "SBS Threshold of a fiber with Brillouin frequency shift distribution", JLT 14, 50-57 (1996).

[2] J. H. Lee et al., "Investigation of Brillouin effects in small-core holey optical fiber: lasing and scattering" Opt. Lett. 27, 927-929, (2002).

[3] K. Furusawa et al., "Correlation between the Brillouin and the structural parameters in holey optical fibers", CLEO May 2004, paper CThHH.

[4] G. Agrawal, Nonlinear fiber optics, 3rd ed . (Academic Press, 2001), Chap.9.

[5] J. H. Lee et al., "Experimental comparison of a Kerr nonlinearity figure of merit including the stimulated Brillouin scattering threshold for state-of-the-art nonlinear optical fibers", Opt. Lett. 30, 1698-1700, (2005). 